\title{
The effects of electron surface interactions in geometrically symmetric capacitive RF plasmas in the presence of different electrode surface materials
}

Cite as: Phys. Plasmas 26, 063505 (2019); https://doi.org/10.1063/1.5094100

Submitted: 27 February 2019 . Accepted: 14 May 2019 . Published Online: 06 June 2019

Jing-Yu Sun (D), De-Qi Wen, Quan-Zhi Zhang (D), Yong-Xin Liu (D), and You-Nian Wang (D)
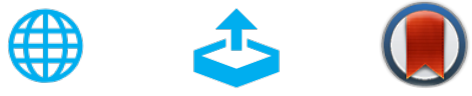

View Online

Export Citation

\section{ARTICLES YOU MAY BE INTERESTED IN}

Numerical studies on plasma parameter modulation of atmospheric pressure dielectric barrier discharge via $200 \mathrm{kHz} / 13.56 \mathrm{MHz}$ dual-frequency excitation

Physics of Plasmas 26, 063504 (2019); https://doi.org/10.1063/1.5091815

\section{Electron kinetics in low-temperature plasmas}

Physics of Plasmas 26, 060601 (2019); https://doi.org/10.1063/1.5093199

Comment on "Symmetry in electron and ion dispersion in 1D Vlasov-Poisson plasma" [Phys. Plasmas 25, 112102 (2018)]

Physics of Plasmas 26, 064701 (2019); https://doi.org/10.1063/1.5090595

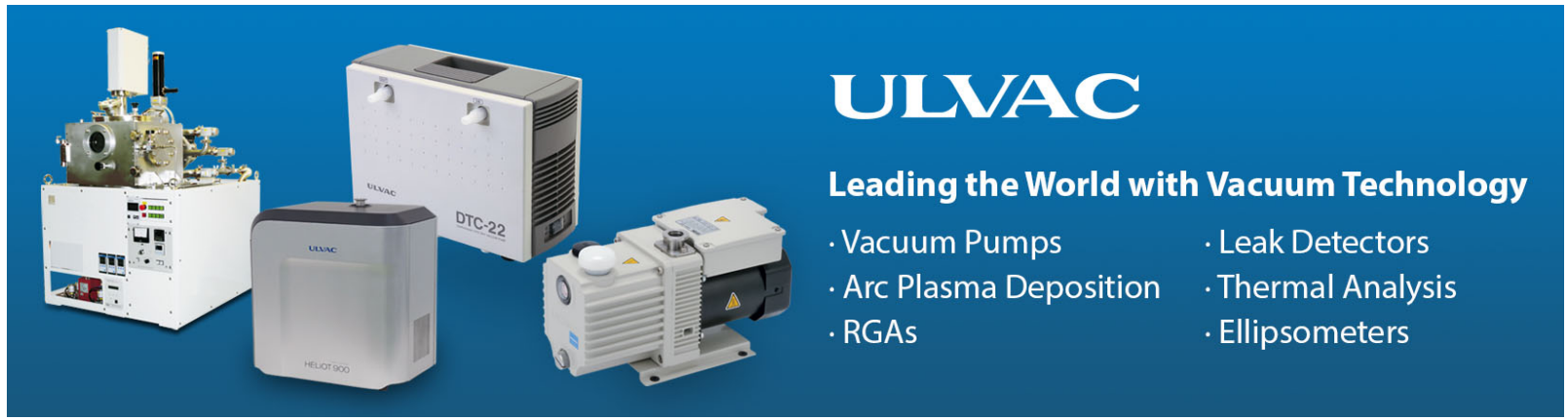




\title{
The effects of electron surface interactions in geometrically symmetric capacitive RF plasmas in the presence of different electrode surface materials
}

Cite as: Phys. Plasmas 26, 063505 (2019); doi: 10.1063/1.5094100

Submitted: 27 February 2019 - Accepted: 14 May 2019 .

Published Online: 6 June 2019

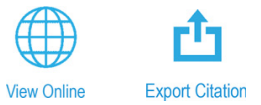

Jing-Yu Sun, (D) De-Qi Wen, ${ }^{2}$ Quan-Zhi Zhang, ${ }^{3}$ (D) Yong-Xin Liu, ${ }^{1}$ (D) and You-Nian Wang ${ }^{1, a)}$ (D)

\author{
AFFILIATIONS \\ ${ }^{7}$ School of Physics, Dalian University of Technology, Dalian 116024, People's Republic of China \\ ${ }^{2}$ Department of Electrical and Computer Engineering, Michigan State University, East Lansing, Michigan 48824, USA \\ ${ }^{3}$ Research Group PLASMANT, University of Antwerp, Universiteitsplein 1, B-2610 Antwerp-Wilrijk, Belgium

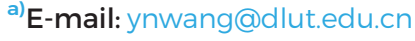

\begin{abstract}
Particle-in-cell/Monte Carlo collision (PIC/MCC) simulations are performed to investigate the asymmetric secondary electron emission (SEE) effects when electrons strike two different material electrodes in low pressure capacitively coupled plasmas (CCPs). To describe the electron-surface interactions, a realistic model, considering the primary electron impact energy and angle, as well as the corresponding surface property-dependent secondary electron yields, is employed in PIC/MCC simulations. In this model, three kinds of electrons emitted from the surface are considered: (i) elastically reflected electrons, (ii) inelastically backscattered electrons, and (iii) electron induced secondary electrons (SEs, i.e., $\delta$-electrons). Here, we examined the effects of electron-surface interactions on the ionization dynamics and plasma characteristics of an argon discharge. The discharge is driven by a voltage source of $13.56 \mathrm{MHz}$ with amplitudes in the range of 200-2000 V. The grounded electrode material is copper (Cu) for all cases, while the powered electrode material is either $\mathrm{Cu}$ or silicon dioxide $\left(\mathrm{SiO}_{2}\right)$. The simulations reveal that the electron impact-induced SEE is an essential process at low pressures, especially at high voltages. Different electrode materials result in an asymmetric response of SEE. Depending on the instantaneous local sheath potential and the phase of the SEE, these SEs either are reflected by the opposite sheath or strike the electrode surface, where they can induce $\delta$-electrons upon their residual energies. It is shown that highly energetic $\delta$-electrons contribute significantly to the ionization rate and a self-bias forms when the powered electrode material is assumed to be made of $\mathrm{SiO}_{2}$. Complex dynamics is observed due to the multiple electron-surface interaction processes and asymmetric yields of SEs in CCPs.
\end{abstract}

Published under license by AIP Publishing. https://doi.org/10.1063/1.5094100

\section{INTRODUCTION}

Low-pressure capacitively coupled plasmas (CCPs) are of vital importance for extensive applications in microelectronic device manufacturing. ${ }^{1-3}$ When the capacitive discharges are sustained in a steady state, plasma particles including electrons and ions, as well as fast weight neutrals, flow toward the bound substrate or metal electrodes. Thus, the material properties of the electrode could be modified. Meanwhile, the energetic particles striking the surface will induce secondary electron emission (SEE), which, in turn, affects the discharge states. Therefore, the interactions between plasma and the surface are attracting more and more interest in both academia and industries. The particle-in-cell/Monte Carlo collision (PIC/MCC) method employs the fundamental equations without much approximation, allowing us to retain most of the physics. ${ }^{4}$ Thus, it is an efficient measure to investigate the complex physics of plasma discharges. ${ }^{5-7}$ In order to investigate the effect of SEE on the plasma physics, simplified models were originally used in PIC/MCC simulations, i.e., the accelerated positive ions through the sheath induce a constant SEE yield, which was independent of the incident particle energy and angle. ${ }^{7-1}$ The importance of the incident particle energies and surface conditions on SEE was explored in recent studies of low-pressure argon CCPs. ${ }^{15-20}$ Daksha et al. ${ }^{19}$ found that the heavy particle energydependent SEE has a negative influence on the separate control of ion properties compared to the constant SEE yield model for ion bombardment. Besides conducting PIC/MCC simulations in capacitive oxygen discharges, Hannesdottir and Gudmundsson ${ }^{18}$ revealed that 
SEs induced by heavy particles $\left(\mathrm{O}^{+}\right.$ions, $\mathrm{O}_{2}^{+}$ions, $\mathrm{O}$ atoms, and $\mathrm{O}_{2}$ molecules) have a significant impact on the electron density profile and the sheath width when an energy-dependent SEE yield is applied.

In addition to the fast heavy particle-induced SEs, electrons can be heated to be highly energetic by an expanding sheath and then pass through the plasma bulk without any impacts with neutrals to approach the opposite electrode at low pressures and high driving voltages. When these highly energetic electrons overcome the potential drop of the opposite sheath and strike the surface, a significant SEE even with the emission yield larger than one can occur. As a result, the characteristic of the plasma is affected. To describe such an electroninduced SEE process, a realistic SEE model for electrons was ever proposed by Gopinath et al., ${ }^{21}$ considering the incident electron energy and angle, as well as the corresponding secondary electron emission coefficients (SEECs) based on surface properties. In this realistic model, the total yield of SEs due to the electron impact consists of three components: (i) elastically reflected electrons (el.-electrons), (ii) inelastically backscattered electrons (inel.-electrons), and (iii) electroninduced SEs ( $\delta$-electrons). Recently, Horváth et al. ${ }^{22}$ used this realistic model in PIC/MCC simulations and observed significantly enhanced ionization dynamics compared to the results obtained from a simplified model for the electron-surface interactions. Of which, the plasma is sustained at a pressure as low as $0.5 \mathrm{~Pa}$ and bound by two $\mathrm{SiO}_{2}$ electrodes with a gap of $6.7 \mathrm{~cm}$. The simulation results clearly revealed the ionization mechanism of SEs induced by primary electrons and its impact on the discharge parameters. The latest work of Horváth et $a l^{23}$ studied the influence of $\delta$-electrons on the ionization dynamics and plasma characteristics at various pressures (between $0.5 \mathrm{~Pa}$ and $3.0 \mathrm{~Pa}$ ) and voltage amplitudes, assuming different secondary electron (SE) yields induced by ions in a single-frequency (SF) source of $13.56 \mathrm{MHz}$ argon discharges. The effect of $\delta$-electrons on the ionization dynamics is the most remarkable at low pressures, high voltage amplitudes, and high values of the $\gamma$ coefficients.

However, in realistic semiconductor manufacturing, a wafer is usually placed on one electrode of the CCP chamber, and the other electrode is a metal material and grounded. Actually, previous simulation studies usually focused on asymmetric SEE induced by ion bombardment in CCPs. ${ }^{24,25}$ The asymmetric plasma response is produced at relatively high pressures because of direct-current (DC) self-bias induced by asymmetric $\gamma$-electron yields. However, the influence of asymmetric SEE induced by electrons is ignored. Thus, our aim is to have an insight into the effects of asymmetric electrode materialinduced SEE on the ionization dynamics and plasma parameters in low-pressure CCPs by performing a PIC/MCC simulation.

In this work, we carry out a detailed investigation of the effects of electron-induced SEE on the plasma parameters, especially the ionization dynamics in CCPs by PIC/MCC simulations. The discharges are operated at a low pressure of $2.0 \mathrm{~Pa}$ in argon. The powered electrode materials are chosen to be $\mathrm{Cu}$ or $\mathrm{SiO}_{2}$. Hence, the corresponding asymmetry in the emission coefficients of the SEs induced by electrons is taken into account. A realistic description of electron-surface interactions, considering the energy and angle of primary electrons, as well as the surface properties, is employed in our PIC/MCC simulations.

This paper is structured in the following way. In Sec. II, a brief description of PIC/MCC simulation and the self-consistent SEE model used in simulations are introduced. The simulation results are presented and discussed in Sec. III. Finally, conclusions are drawn in Sec. IV.

\section{DESCRIPTION OF SIMULATION}

\section{A. PIC/MCC simulation}

The simulations are based on a standard 1D3V (i.e., onedimensional in space and three-dimensional in velocity) electrostatic PIC/MCC method. ${ }^{4,26,27}$ The discharge is sustained between two parallel-plate and infinite electrodes with a gap distance of $4 \mathrm{~cm}$ in argon at $2.0 \mathrm{~Pa}$. In the Monte Carlo collision process, we separately considered elastic, excitation, and ionization collisions for electrons, as well as elastic and charge transfer collisions for $\mathrm{Ar}^{+}$ions. The electron cross-sectional data applied are given in Ref. 28, and the ion cross sections are adopted from Ref. 29. A voltage source $V(t)=V_{0} \cos (2 \pi f t)$ with $f=13.56 \mathrm{MHz}$ was connected with the electrode at the position of $z=0 \mathrm{~cm}$. The plasma particles traced in the simulations are electrons and $\mathrm{Ar}^{+}$ions. We assume that the powered electrode is made of different materials. This assumption leads to an asymmetric yield of SEs emitted from two different electrode surfaces. The opposite electrode is at the position of $z=4 \mathrm{~cm}$ and grounded. As for the ion-induced SEE coefficient, a commonly used parameter $\gamma=0.2^{10,14}$ was assumed in all cases. The assumption for the ion-induced SEE coefficient is simplified since we aim to present the effects of the realistic electron-surface interactions on the discharge characteristics. The initial velocities of the $\gamma$ electrons emitted from the surfaces are sampled from a Maxwellian distribution with an average electron temperature of $2.0 \mathrm{eV}$, and these $\gamma$-electrons are emitted toward the plasma in an isotropic way. The gas temperature is constant, $400 \mathrm{~K}$. The initial electron and $\mathrm{Ar}^{+}$ion temperatures are set to be $2.0 \mathrm{eV}$ and $0.026 \mathrm{eV}$, respectively.

In order to consider the generation of the DC self-bias in the simulation, we count the accumulated electrons and ions, as well as the number of SEs induced by incident electrons and ions at each electrode separately, and adjust the DC self-bias iteratively until the cycle averaged electron and ion fluxes at each electrode are balanced. ${ }^{30}$ This DC self-bias will change the plasma potential and, in turn, affect the SE yields induced by electrons at both electrodes.

Our simulations are performed to study the discharge operated in two different cases. In case A, both electrodes are assumed to be made of $\mathrm{Cu}$; In case $\mathrm{B}$, the powered electrode material is set to be $\mathrm{SiO}_{2}$ and the grounded electrode material is the same as in case A.

\section{B. Electron-surface interaction model}

In our simulations, we include an accurate material specific treatment regarding the electron-surface interactions, which takes into account the energy and angle of primary electrons, as well as the surface properties to determine the partial emission coefficients of different electron-surface interactions. Horváth $e t a .^{22}$ have introduced this realistic model in detail. Here, we just briefly review it. The total yield of SEs, $\sigma$, due to primary electron bombardment is composed of three components: (i) elastically reflected electron yield, $\eta_{\mathrm{el}}$, (ii) inelastically backscattered electron yield, $\eta_{\text {inel, }}$ and (iii) true SE yield, $\delta$. Therefore

$$
\sigma=\eta_{\text {el. }}+\eta_{\text {inel. }}+\delta \text {. }
$$

The corresponding emission coefficients are determined as proposed by Sydorenko. ${ }^{31}$ Vaughan ${ }^{32,33}$ has modeled this empirical formula of the total electron-induced SEECs as follows:

$$
\sigma_{V}=\sigma_{\max } W(w) \text {. }
$$

The energy dependence appears implicitly in the right-hand side of Eq. (2) through 


$$
W(w)=\left[w e^{1-w}\right]^{k},
$$

where the normalized energy $w$ is given by

$$
w=\frac{\varepsilon-\varepsilon_{0}}{\varepsilon_{\max }-\varepsilon_{0}}
$$

and

$$
k= \begin{cases}0.56 & w<1 \\ 0.25 & w \geqslant 1\end{cases}
$$

where $\varepsilon$ is the energy of primary electrons, $\varepsilon_{0}$ is the threshold energy for the emission of electron-induced SEs. $k$ is a curve fit parameter, which was determined by Vaughan ${ }^{32}$ to fit the model. The SE yield normally increases with the incidence angle $\theta(\theta=0$ signifies normal incidence). The incidence angular dependence is illustrated in Ref. 4 for electron-induced SEE. An analytical model of angular dependence was proposed by Vaughan, ${ }^{32}$ which accounts for the variation of $\sigma_{\max }$ and $\varepsilon_{\max }$ with energy. $k_{s}$ is a smoothness factor in order to model the characteristics of the surface, whose value can vary between 0 for rough surfaces and 2 for polished surfaces. This model modifies the values of $\sigma_{\max }$ and $\varepsilon_{\max }$ as

$$
\begin{gathered}
\sigma_{\max }=\sigma_{\max , 0}\left(1+\frac{k_{s}}{2 \pi} \theta^{2}\right), \\
\varepsilon_{\max }=\varepsilon_{\max , 0}\left(1+\frac{k_{s}}{\pi} \theta^{2}\right),
\end{gathered}
$$

where $\varepsilon_{\max , 0}$ is the energy at the maximum yield and $\sigma_{\max , 0}$ is the corresponding yield for normal incidence. The model takes into account the variation of the electron-induced SE yield (i.e., various $\sigma_{\max , 0}$ ) with the energy and angle of incidence as well as with the surface properties via Eqs. (2)-(6). Sydorenko ${ }^{31}$ presented a correction to the total emission coefficient $\sigma_{\mathrm{V}}$ proposed by Vaughan. ${ }^{32}$ In order to take into account the electron elastic reflection process, which has a significant effect due to low primary electron energies, the elastic reflection coefficient, $\eta_{\mathrm{el}}$, is calculated as

$$
\begin{gathered}
\eta_{\mathrm{el} .}=r_{\mathrm{el} .} \sigma_{\mathrm{V}}+ \begin{cases}\eta_{\mathrm{el}, \text { max }} w_{1} e^{1-w_{1}} & \varepsilon_{\mathrm{el} ., 0}<\varepsilon<\varepsilon_{\mathrm{el}, \text { max }} \\
\eta_{\mathrm{el}, \text { max }}\left[1+w_{2}\right] e^{-w_{2}} & \varepsilon>\varepsilon_{\mathrm{el}, \text { max }},\end{cases} \\
w_{1}=\frac{\varepsilon-\varepsilon_{\mathrm{el}, 0}}{\varepsilon_{\mathrm{el} ., \max }-\varepsilon_{\mathrm{el} ., 0}}, \\
w_{2}=\frac{\varepsilon-\varepsilon_{\mathrm{el}, \text { max }}}{\Delta_{\mathrm{el} .}},
\end{gathered}
$$
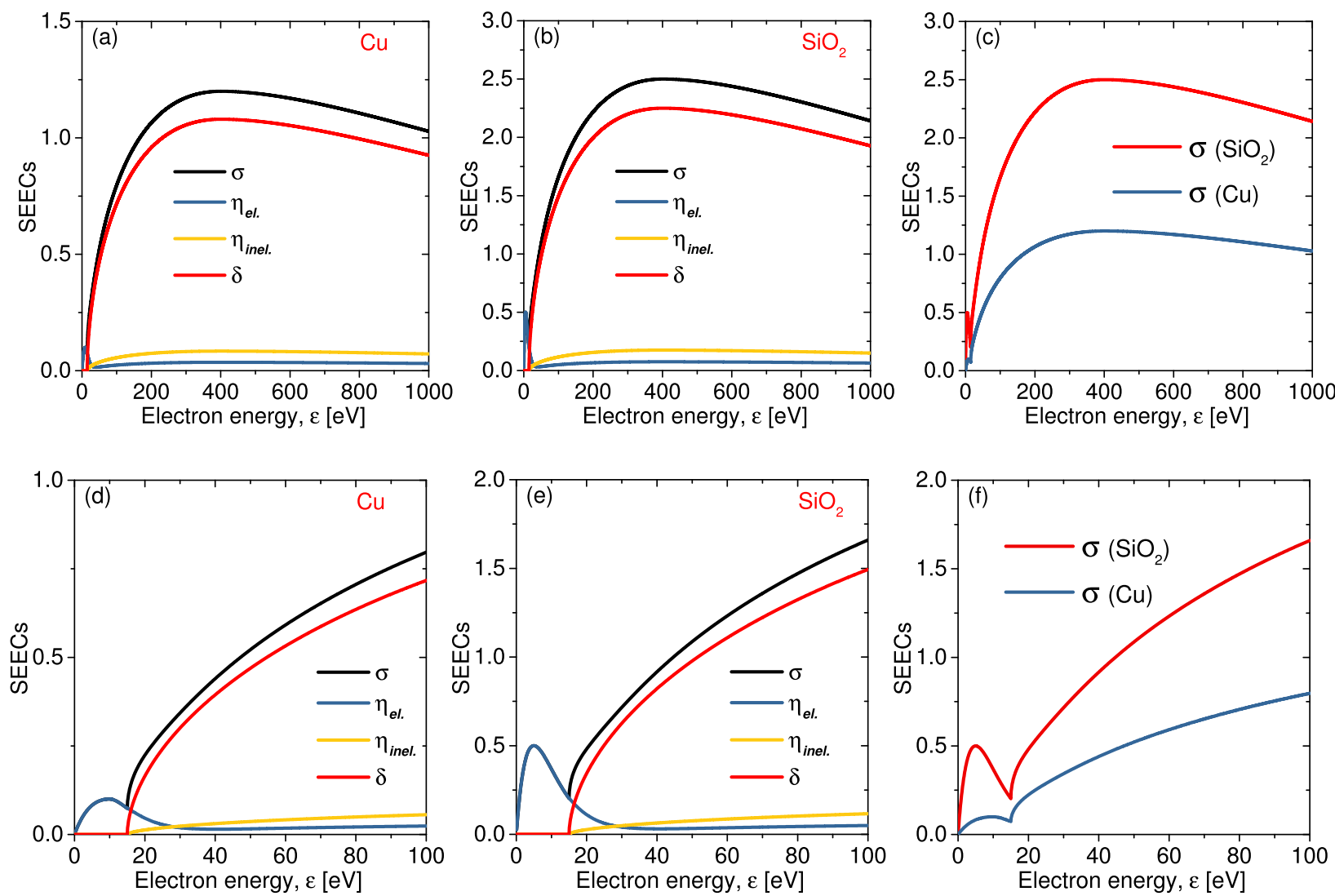

FIG. 1. The total electron-induced emission coefficient $\sigma$ and the partial emission coefficients of the elastic reflection $\eta_{\mathrm{el}}$, inelastic backscattering $\eta_{\text {inel., }}$ and electron-induced SEE $\delta$ as a function of the incident electron energy $\varepsilon$, obtained for normal incidence $(\theta=0)$ for $\mathrm{SiO}_{2}$ and Cu electrode surfaces. 
TABLE I. Typical secondary electron emission parameters for $\mathrm{SiO}_{2}$ and $\mathrm{Cu}$.

\begin{tabular}{|c|c|c|c|c|}
\hline \multirow[b]{2}{*}{ No. } & \multirow[b]{2}{*}{ Parameter } & \multirow[b]{2}{*}{ Description } & \multicolumn{2}{|c|}{ Value } \\
\hline & & & $\mathrm{SiO}_{2}$ & $\mathrm{Cu}$ \\
\hline 1. & $\varepsilon_{0}$ & The threshold energy for electron-induced $\delta$-electron emission & $15 \mathrm{eV}$ (Ref. 22) & $15 \mathrm{eV}$ (Ref. 21) \\
\hline 2. & $\varepsilon_{\max , 0}$ & The energy of primary electrons at the maximum emission & 400 eV (Ref. 35) & $400 \mathrm{eV}$ (Ref. 21) \\
\hline 3. & $\sigma_{\max , 0}$ & The maximum emission at normal incidence & 2.5 (Ref. 35) & 1.2 (Ref. 21) \\
\hline 4. & $k_{s}$ & Smoothness factor of the surface & 1 & 1 \\
\hline 5. & $\varepsilon_{\mathrm{el} .0}$ & The threshold energy for elastic reflection & $0 \mathrm{eV}$ (Ref. 36) & 0 eV (Ref. 36) \\
\hline 6. & $\varepsilon_{\mathrm{el}, \text { max }}$ & The energy of primary electrons at the maximum elastic reflection & $5 \mathrm{eV}$ (Ref. 34) & $10 \mathrm{eV}$ (Ref. 34) \\
\hline 7. & $\eta_{\text {el.,max }}$ & The maximum of the elastic reflection & 0.5 (Ref. 34) & 0.1 (Ref. 34) \\
\hline 8. & $\Delta_{\mathrm{el}}$ & Control parameter for the decay of $\eta_{\mathrm{el}}$. & 5 (Ref. 36) & 5 (Ref. 36) \\
\hline 9. & $r_{\mathrm{el}}$ & Portion of elastically reflected electrons at high energies & 0.03 (Ref. 21) & 0.03 (Ref. 21) \\
\hline 10. & $r_{\text {inel. }}$ & Portion of inelastically reflected electrons at high energies & 0.07 (Ref. 21) & 0.07 (Ref. 21) \\
\hline
\end{tabular}

where $\varepsilon_{\mathrm{el}, 0}$ is the threshold energy for the elastic reflection and $\Delta_{\mathrm{el}}$. is used to control the decay of $\eta_{\mathrm{el}}$. as a function of incident electron energies $\varepsilon$, when $\varepsilon$ is higher than the energy of primary electrons at the maximum elastic reflection $\varepsilon_{\mathrm{el} ., \mathrm{max}}$. At energies above $\varepsilon_{\mathrm{el} ., 0}$, the el.-electrons typically comprise about $3 \%$ of the total ejected electrons, ${ }^{4,21}$ and they have the full incident energy. The inel.-electrons, comprising about $7 \%$ of the ejected electrons, ${ }^{4,21}$ have energy between zero and the incident energy. The $\delta$-electrons, comprising about $90 \%$ of the ejected electrons, and initial velocities of the $\delta$-electrons are taken as a Maxwell-Boltzmann distribution at a temperature of tens of $\mathrm{eV}$.

$r_{\mathrm{el}}$. controls the portion of the el.-electrons in the total electroninduced SEs for high energies (secondaries with energy $>\varepsilon_{0}$ ). The inelastic reflection coefficient, $\eta_{\text {inel, }}$, is determined as

$$
\eta_{\text {inel. }}=r_{\text {inel. }} \sigma_{\mathrm{V}},
$$

where $\eta_{\text {inel. }}$ controls the portion of the inel.-electrons in the total electron-induced SE flux. Finally, the yield of true secondaries $\delta$ is obtained as

$$
\delta=\left(1-r_{\text {el. }}-r_{\text {inel. }}\right) \sigma_{\mathrm{V}}
$$

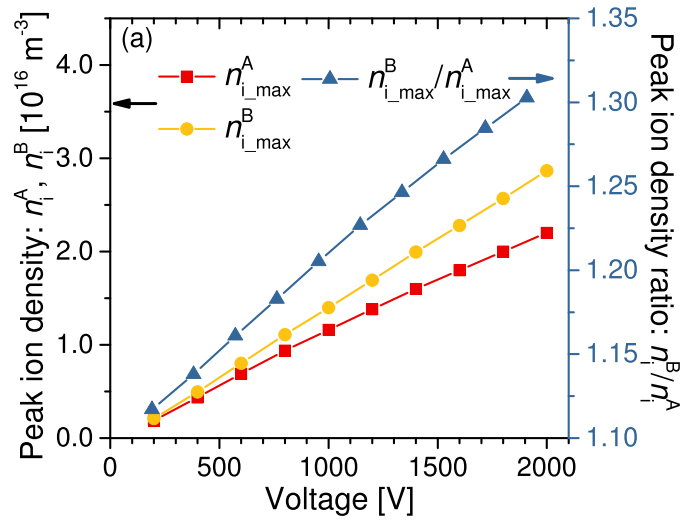

Therefore, $\delta$ is set to 0.9 to reflect the portion of the $\delta$-electrons in the ejected SEs induced by electrons. Due to the correction term in the equation, the total emission coefficient $\sigma$ differs from $\sigma_{\mathrm{V}}$ at low incident energies.

Figure 1 shows the total electron-induced SEE coefficient $\sigma$ and the partial emission coefficients of the elastic reflection $\eta_{\mathrm{el}}$, inelastic backscattering $\eta_{\text {inel, }}$, as well as electron-induced SEE $\delta$ as a function of the incident electron energy $\varepsilon$, at normal incidence for $\mathrm{Cu}$ [Figs. 1 (a) and 1(d)] and $\mathrm{SiO}_{2}$ [Figs. 1(b) and 1(e)] electrode surfaces by fitting the above empirical formulas. The bottom row in Fig. 1 is the enlargement of the corresponding figures in the top row of Fig. 1 in the range of electron energies from 0 to $100 \mathrm{eV}$. The parameters used in the realistic model of the electron-surface processes for $\mathrm{SiO}_{2}$ and $\mathrm{Cu}$ are listed in Table I. ${ }^{22}$ As shown in Fig. 1(c), the general shape of $\sigma_{\mathrm{V}}$ has a similar trend for different surface materials, and $\sigma_{\mathrm{V}}$ increases rapidly with increasing incident electron energy $\varepsilon$. It first reaches a maximum value of $\sigma_{\max , 0}$ at a primary electron energy of $\varepsilon_{\max , 0}$ and then decreases slowly as primary energies increase. The values of $\sigma_{\text {max }, 0}$ vary over a wide range for different materials, and $\sigma_{\max , 0}$ is set to 1.2 for $\mathrm{Cu}$ and 2.5 for $\mathrm{SiO}_{2}$, respectively. As for the elastic reflection yield $\eta_{\mathrm{el}}$, it is generally higher at low energies for

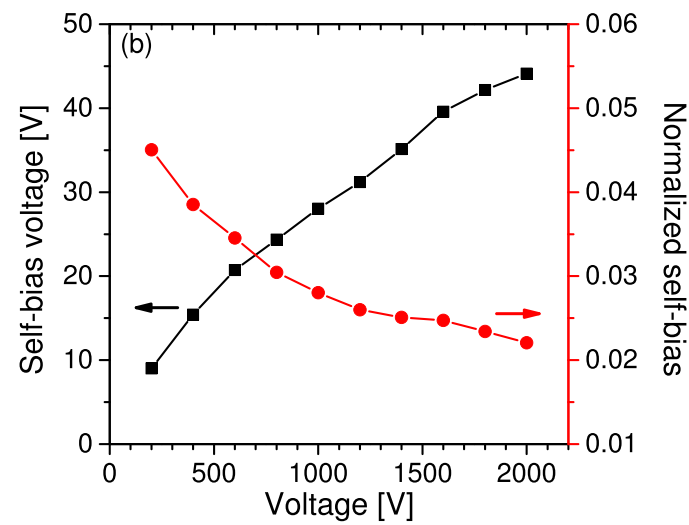

FIG. 2. Left: The central ion density obtained from case $\mathrm{A}$ and case $\mathrm{B}, n_{\mathrm{i}-\max }^{\mathrm{A}}$ and $n_{\mathrm{i} \text { max }}^{\mathrm{B}}$ (left vertical scale), and the density ratio $n_{\mathrm{i}-\max }^{\mathrm{B}} / n_{\mathrm{i}-\max }^{\mathrm{A}}$ (right vertical scale) as a function of the driving voltage amplitude, $V_{0}$; Right: The plot of the DC self-bias obtained from case $B$ as a function of the driving voltage amplitude, $V_{0}$. Discharge conditions: $d=4.0 \mathrm{~cm}$, $f=13.56 \mathrm{MHz}$, and $p=2.0 \mathrm{~Pa}$. In case A, both electrode materials are $\mathrm{Cu}$; In case $\mathrm{B}$, the powered electrode material is $\mathrm{SiO}_{2}$ and the grounded electrode material is $\mathrm{Cu}$. 

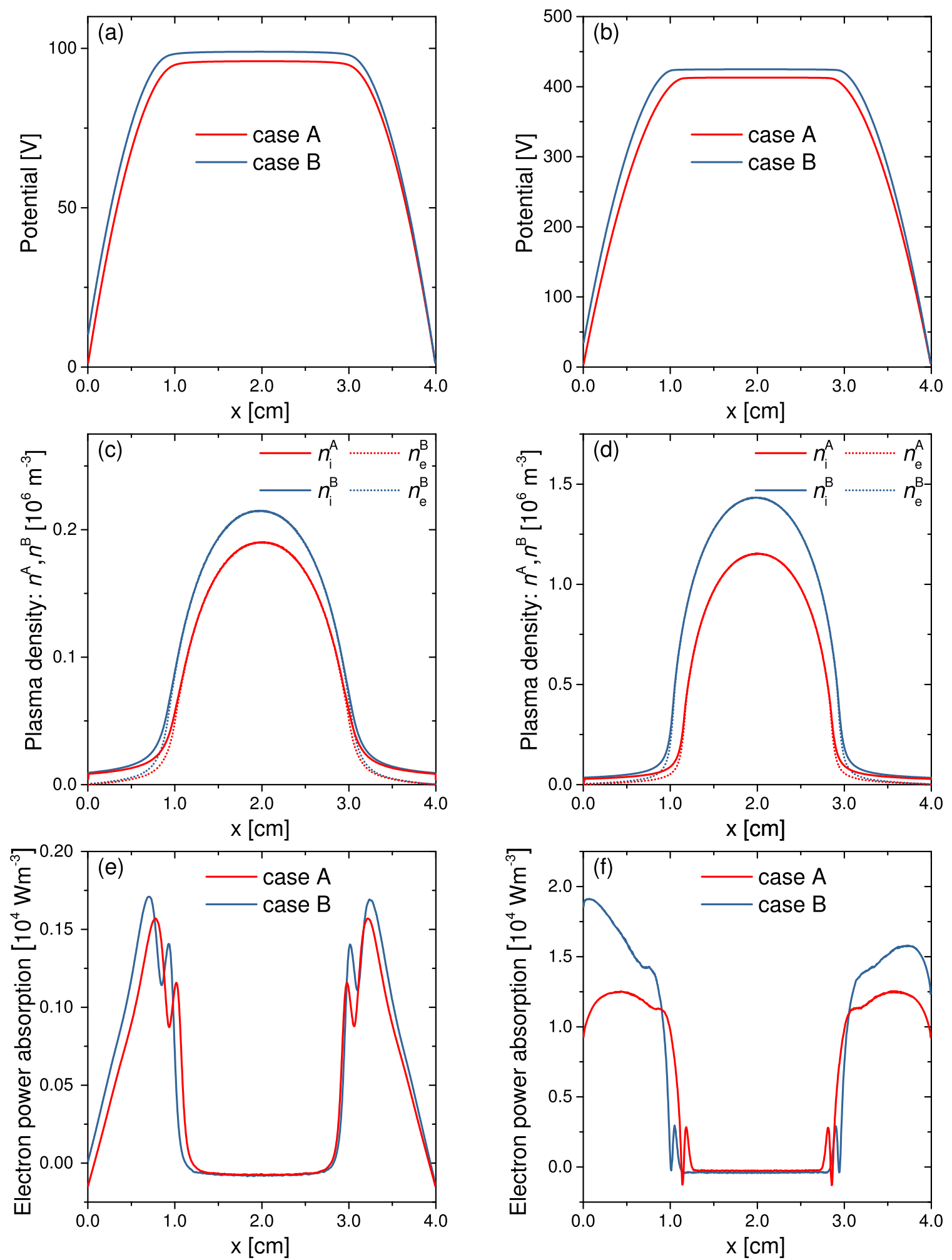

FIG. 3. Time-averaged spatial profiles of the plasma potential (first row), the charged particle density (second row), and the electron power absorption (third row) obtained from both cases at $V_{0}=200 \mathrm{~V}$ (left column) and $V_{0}=1000 \mathrm{~V}$ (right column), respectively. Discharge conditions: $d=4.0 \mathrm{~cm}, f=13.56 \mathrm{MHz}$, and $p=2.0 \mathrm{~Pa}$. 
dielectrics than that for metals. ${ }^{34}$ We thus assume that the elastic reflection yield $\eta_{\text {el. }}$ rapidly increases up to 0.5 at $5 \mathrm{eV}$ for $\mathrm{SiO}_{2}$ and 0.1 at $10 \mathrm{eV}$ for $\mathrm{Cu}$ in this model. That is, the total SE yield $\sigma$ of $\mathrm{Cu}$ is always lower than that of $\mathrm{SiO}_{2}$ for all primary electrons.

\section{RESULTS AND DISCUSSION}

\section{A. Effects of SEs on plasma parameters}

Figure 2(a) shows the ion density of case A (both electrode materials are set to be made of $\mathrm{Cu}$ ), $n_{\mathrm{i} \_ \text {max }}^{\mathrm{A}}$, and case $\mathrm{B}$ (the powered electrode material is set to be $\mathrm{SiO}_{2}$, and the grounded electrode material is set to be $\mathrm{Cu}$ ), $n_{\mathrm{i} \_ \text {max }}^{\mathrm{B}}$, in the center of the discharge chamber, as a function of the driving voltage amplitude $V_{0}$, at a pressure of $p=2.0 \mathrm{~Pa}$, an electrode gap of $d=4.0 \mathrm{~cm}$, and a driving frequency of $f=13.56 \mathrm{MHz}$ (left vertical scale). The driving voltages are adjusted from $200 \mathrm{~V}$ to $2000 \mathrm{~V}$. One can see that the ion density increases linearly with rising $V_{0}$ in both cases at a given pressure. As expected, the plasma density obtained from case $\mathrm{B}$ is higher compared to that obtained from case A for all $V_{0}$ due to higher electron-induced SEECs
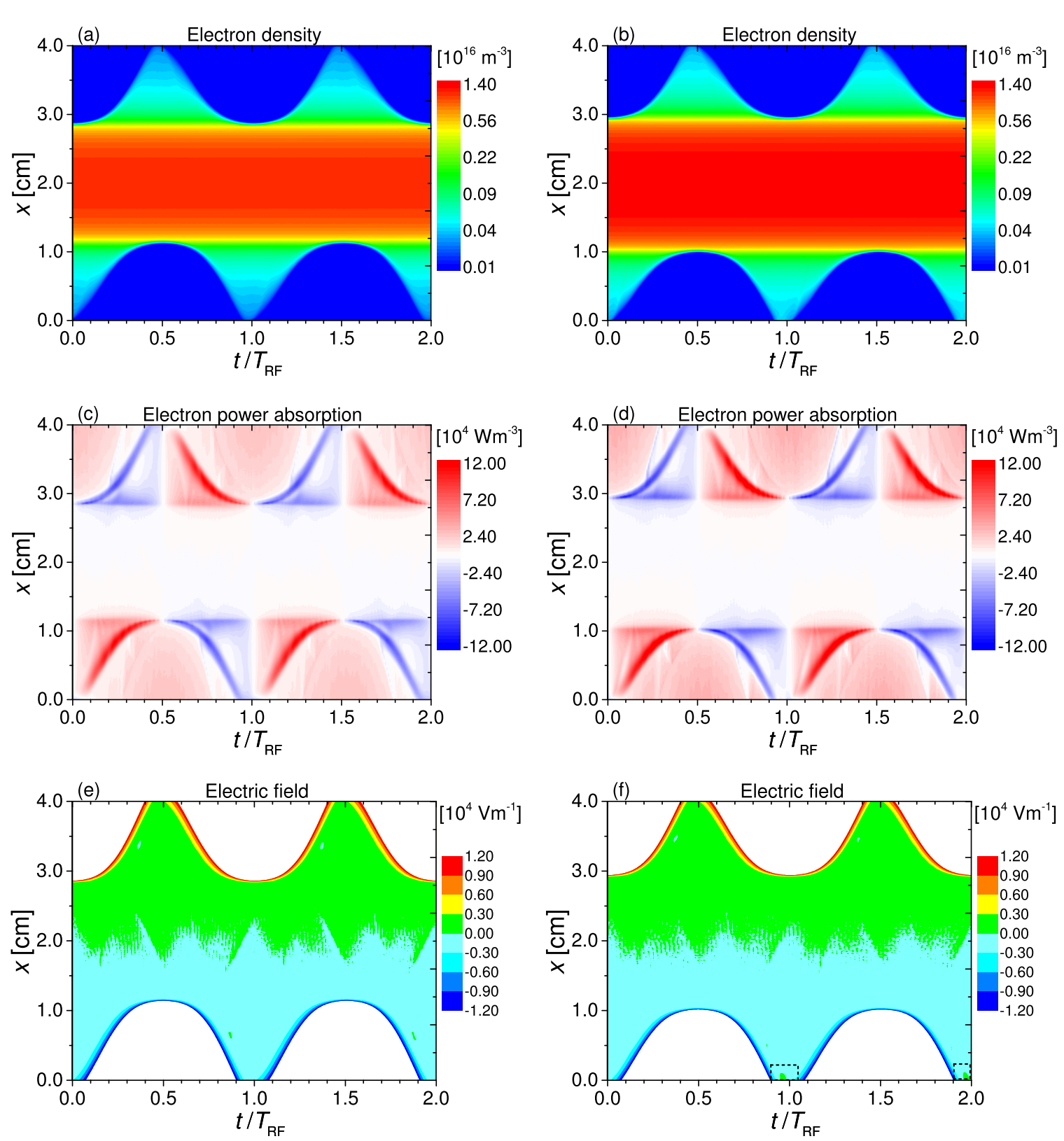

FIG. 4. Spatiotemporal distributions of electron density, of which color scales are logarithmic and cover two orders of magnitude (first row), electron power absorption (second row), and the electric field (third row) obtained from case A (left column) and case B (right column). Discharge conditions: $d=4.0 \mathrm{~cm}, f=13.56 \mathrm{MHz}, p=2.0 \mathrm{~Pa}$, and $V_{0}=1000 \mathrm{~V}$. The dashed rectangles in Fig. $4(f)$ show the regions of field reversal. 
in case B. This indicates that the effect of electron-induced SEE on the plasma density is pronounced, in spite of the fact that only the powered electrode material is $\mathrm{SiO}_{2}$. On the right vertical scale of Fig. 2(a), the ratio of the central ion densities, $n_{\mathrm{i}_{-} \max }^{\mathrm{B}} / n_{\mathrm{i} \_ \text {max }}^{\mathrm{A}}$, is given as a function of $V_{0}$. It reveals that relatively higher plasma density is obtained in case $\mathrm{B}$ as $V_{0}$ increases. At the highest voltage amplitude of $V_{0}=2000 \mathrm{~V}$, a maximum density ratio, $n_{\mathrm{i} \_ \text {max }}^{\mathrm{B}} / n_{\mathrm{i} \_ \text {max }}^{\mathrm{A}}$, of about 1.3 is obtained.

The DC self-bias as a function of $V_{0}$ for case $\mathrm{B}$ is presented in Fig. 2(b). The increasing voltage leads to a more pronounced asymmetry in the SEE flux emitted from two electrodes in case B, resulting in an increase in DC self-bias. For an argon discharge at a low pressure of 2.0 $\mathrm{Pa}$, the electron mean free path $\lambda=\left(n_{g} \sigma\right)^{-1}$ is comparable to the discharge scale, and hence, the electron dynamics are in the nonlocal regime. ${ }^{37}$ Consequently, the DC self-bias caused by asymmetric SEECs is lower than expected. ${ }^{25}$ Note that the DC self-bias is normalized by $V_{0}$ on the right vertical scale. Since high voltage enhances the nonlocal properties of electrons, the normalized DC self-bias decreases with increasing $V_{0}$.

Time-averaged spatial profiles of the plasma potential, the charged particle densities, and the electron power absorption $\left(P_{\mathrm{e}}\right.$ $\equiv\left\langle J_{\mathrm{e}} \cdot E\right\rangle$ ) for both cases are shown in Fig. 3 for $V_{0}=200 \mathrm{~V}$ (left column) and $V_{0}=1000 \mathrm{~V}$ (right column), respectively. For case A, the potential profiles are symmetric as expected, whereas the sheath voltage division turns to be asymmetric and a DC self-bias forms at the powered electrode for case B. Because of the higher SE flux at the powered electrode, a lower voltage drop is needed here to balance the electron and ion currents. The results obtained from case B give nearly symmetric profiles of charged particle density due to low DC self-bias. However, the peak densities of charged particles obtained from case B are higher than those obtained from case A for both driving voltages, and the increase in peak densities is more pronounced at a high driving voltage amplitude. The electron power absorption mainly takes place in the sheath regions, and the double peak structure at the expansion sheath edge is caused by the acceleration of electrons under the effect of the ambipolar field. ${ }^{38}$ An asymmetric spatial profile is found in different sheath regions for case B. Although the potential drop of the sheath is lower at the powered electrode (shown in the top row of Fig. 3), the peak of the power absorption is significantly enhanced adjacent to the powered electrode (shown in the bottom row of Fig. 3). It can be understood that the high fluxes of SEs at the powered electrode [see Fig. 9(d)] lead to significant current densities and further enhance the electron power absorption near the powered electrode at a high driving voltage of $V_{0}=1000 \mathrm{~V}$, while the profiles of electron power absorption can hardly be altered at low $V_{0}$.

In order to further explore the effects of electron-induced SEE on plasma parameters at high driving voltages, the spatiotemporal distributions of the key discharge parameters are presented. Figure 4 shows the spatiotemporal distributions of the electron density (first row), the electron power absorption (second row), and the electric field (third row) obtained from case A (left column) and case B (right column) within two RF periods, respectively. Comparing the results obtained from case $\mathrm{A}$ and case $\mathrm{B}$, we can find that the asymmetric discharge caused by different electrode materials has been remarkably enhanced at low pressures and high voltages. In case B [shown in Fig. 4(f)], the generation of an electric field reversal at the powered electrode during sheath collapse has been observed. The regions of field reversal are indicated by dashed rectangles in Fig. 4(f). Similar results were also obtained in Ref. 22. The reversed electric field is generated to balance cycle averaged electron and ion fluxes at the powered electrode. In order to compensate the ion flux, a reversed electric field must be generated to pull back some of the SEs to the powered electrode, where they can be absorbed in a certain probability. ${ }^{39}$

\section{B. Heating mechanism of SEs}

Figure 5 shows the spatiotemporal distributions of the total ionization rate within two RF periods for case $\mathrm{A}$ and case $\mathrm{B}$ at $V_{0}$ $=1000 \mathrm{~V}$, respectively. The ionization is dominated by the electrons that get accelerated at expanding sheath edges in both cases. At the beginning of the RF period, we can clearly observe that a beam of highly energetic electron is generated by the expanding sheath in both cases, which propagates through the entire bulk and induces ionization until it hits the opposing sheath. The trajectory of the reflected beam is clearly visible in Fig. 5(b) and indicated by arrows. Multiple separate beams in the spatiotemporal distributions of the ionization rate can be observed. A strong beam is generated at the expanding sheath edge, and some of these highly energetic electrons are bounced by the opposite sheath in the collapse phase again. This dynamic leads to the formation of the low-energy beam in the opposite direction. In contrast to case B,
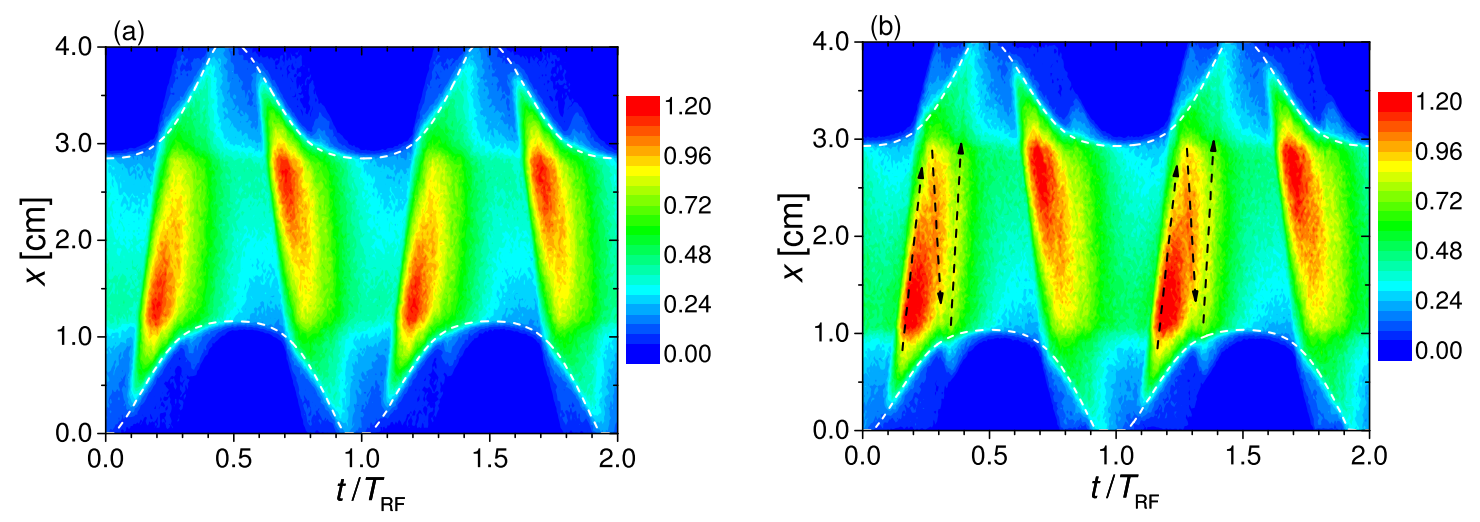

FIG. 5. Spatiotemporal distributions of the total ionization rate within two RF periods obtained from case A (a) and case B (b). Discharge conditions: $d=4.0 \mathrm{~cm}$, $f=13.56 \mathrm{MHz}, p=2.0 \mathrm{~Pa}$, and $V_{0}=1000 \mathrm{~V}$. The sheath edges are defined by $\left|E_{z}\right|=5000 \mathrm{Vm}^{-1}$. The color bars are given in units of $10^{21} \mathrm{~m}^{-3} \mathrm{~s}^{-1}$. 
this reflected electron beam is not obvious in case A. This implies that the low-energy beam is mainly composed of SEs, especially $\delta$-electrons. The speculation is further confirmed by the results in Fig. 6. These highly energetic electron beams can be heated several times by multiple interactions with the sheaths at both electrodes and contribute effective ionization. At a low pressure of $2.0 \mathrm{~Pa}$ and high driving voltages, a number of highly energetic electrons traverse the plasma bulk before transferring their energy through inelastic collisions with the background gas and reach the opposite sheath edge. Consequently, these electrons with high energies could overcome the sheath potential drop to bombard the substrate surfaces and induce SEE.

In the simulations, we distinguish different groups of electrons in order to more clearly understand the influence of SEs on ionization dynamics. $^{22,23}$ Figure 6 shows the individual contribution of bulk electrons (electrons generated by electron or ion impact ionization), $\sigma$-electrons (which comprises all the three components of electron-
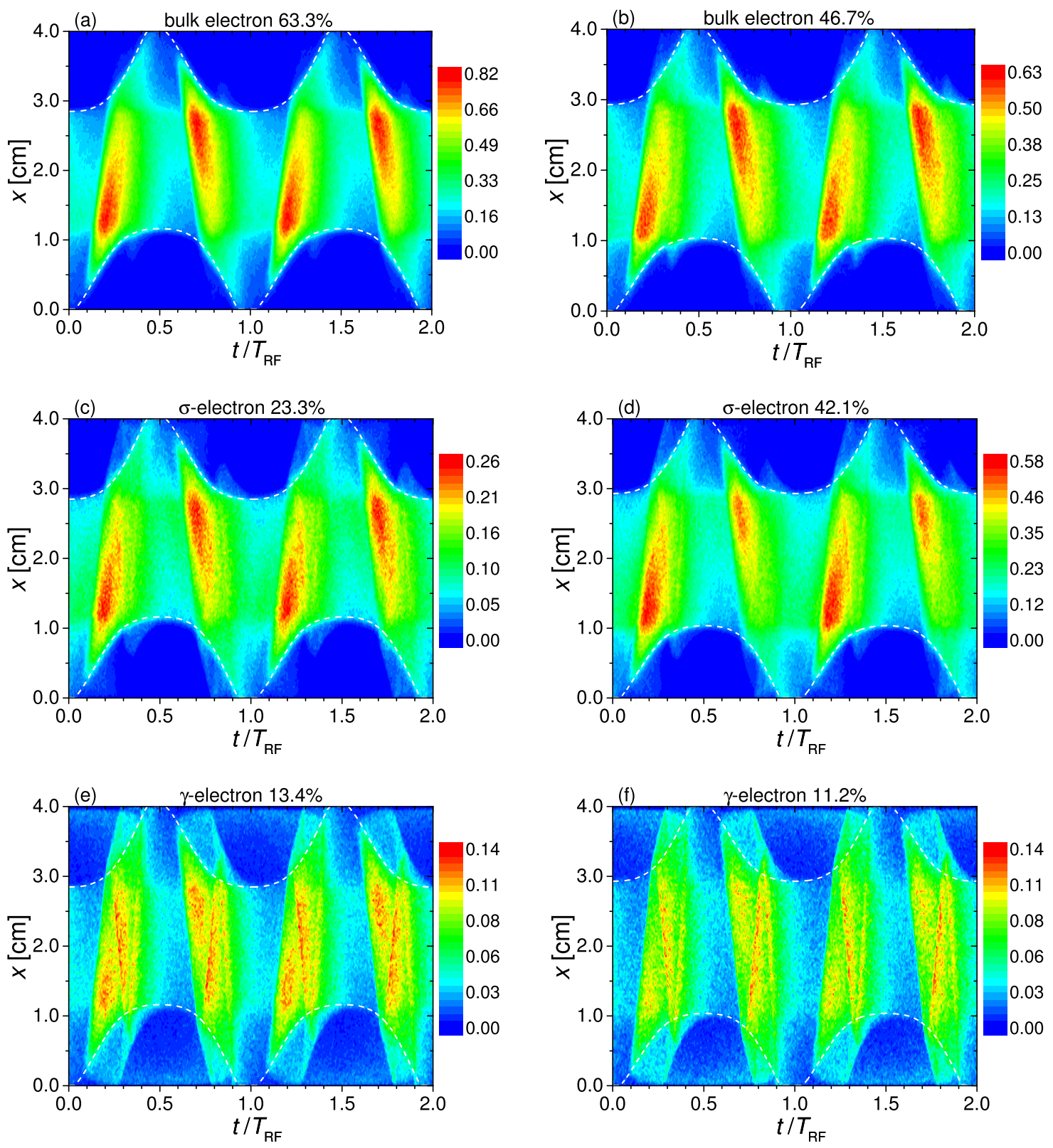

FIG. 6. Spatiotemporal distributions of the contributions of bulk electrons (first row), $\sigma$-electrons (second row), and $\gamma$-electrons (third row) to the total ionization rate (shown in Fig. 5) obtained from case A (left column) and case B (right column). Discharge conditions: $d=4.0 \mathrm{~cm}, f=13.56 \mathrm{MHz}, p=2.0 \mathrm{~Pa}$, and $V_{0}=1000 \mathrm{~V}$. The sheath edges are defined by $\left|E_{z}\right|=5000 \mathrm{Vm}^{-1}$. The color bars are given in units of $10^{21} \mathrm{~m}^{-3} \mathrm{~s}^{-1}$. 
induced SEs), and $\gamma$-electrons to the total ionization rate (see Fig. 5) within two RF periods based on case A (left column) and case B (right column). By comparing the results of case A with those of case $\mathrm{B}$, it can be found that there is a crucial difference in the contributions of different groups of SEs to the total ionization rate. In case B, $\sigma$-electrons play a vital role in the ionization dynamics. $42.1 \%$ of the total ionization rate is directly generated by these SEs at $V_{0}=1000$, while the contribution of $\gamma$-electrons to the total ionization rate is only $11.2 \%$. The remaining $46.7 \%$ of the total ionization rate is generated by bulk electrons. Namely, the contribution of $\sigma$-electrons and bulk electrons to the total ionization rate is comparable in case B at $V_{0}=1000 \mathrm{~V}$. However, bulk electrons dominate ionization in case A, as $63.3 \%$ of the total ionization rate is induced by bulk electrons. The portion of the total ionization rate caused by $\sigma$-electrons and $\gamma$-electrons is only $23.3 \%$ and $13.4 \%$, respectively. Actually, quite a number of bulk electrons are directly generated by $\delta$-electron impact ionization. This means that the $\delta$-electrons indirectly contribute to an even higher ionization rate. It can be seen from Fig. 6 that bulk electrons get accelerated during the phase of sheath expanding and penetrate into the plasma bulk. These bulk electron beams are damped rapidly by colliding with the background gas and efficiently confined inside the bulk region. $\sigma$-electron beams are launched before the sheath completely collapses at both electrodes and accelerated by the residual sheath voltage (as shown in
Fig. 9). These highly energetic $\sigma$-electron beams can traverse the plasma bulk and hit the opposite sheath, where some of them are reflected back into the bulk and form some low-energy beams. The $\gamma$-electrons emitted at the local electrode and accelerated by the high instantaneous sheath voltage can also overcome the sheath residual potential at the opposite electrode. Depending on the instantaneous local sheath potential, they either are reflected back into the bulk or hit the opposite electrode with high energies and generate a number of $\delta$-electrons. ${ }^{22}$ The SEs induced by electrons and ions can be bounced multiple times by time-varying sheaths and generate additional ionization.

Figure 7 presents the individual contribution of reflected electrons (including el.- and inel.-electrons) and $\delta$-electrons to the total ionization rate (see Fig. 5). The reflected electron beams launch shortly during sheath collapse at two electrodes (see the bottom row in Fig. 9). These reflected electron beams are accelerated by locally expanding sheath and damped rapidly due to collisions, which are similar to bulk electron beams. In both cases, $\delta$-electrons with high energies contribute more ionization due to multiple reflections. Furthermore, the ionization rate induced by $\delta$-electron beams emitted by the powered electrode is stronger than that emitted by the grounded electrode in case $\mathrm{B}$. Since the $\delta$ coefficients are higher by a factor larger than two for $\mathrm{SiO}_{2}$ compared to $\mathrm{Cu}$ (shown as Fig. 1), this significant difference in $\delta$ coefficients results in an asymmetric flux of $\delta$-electrons [shown as
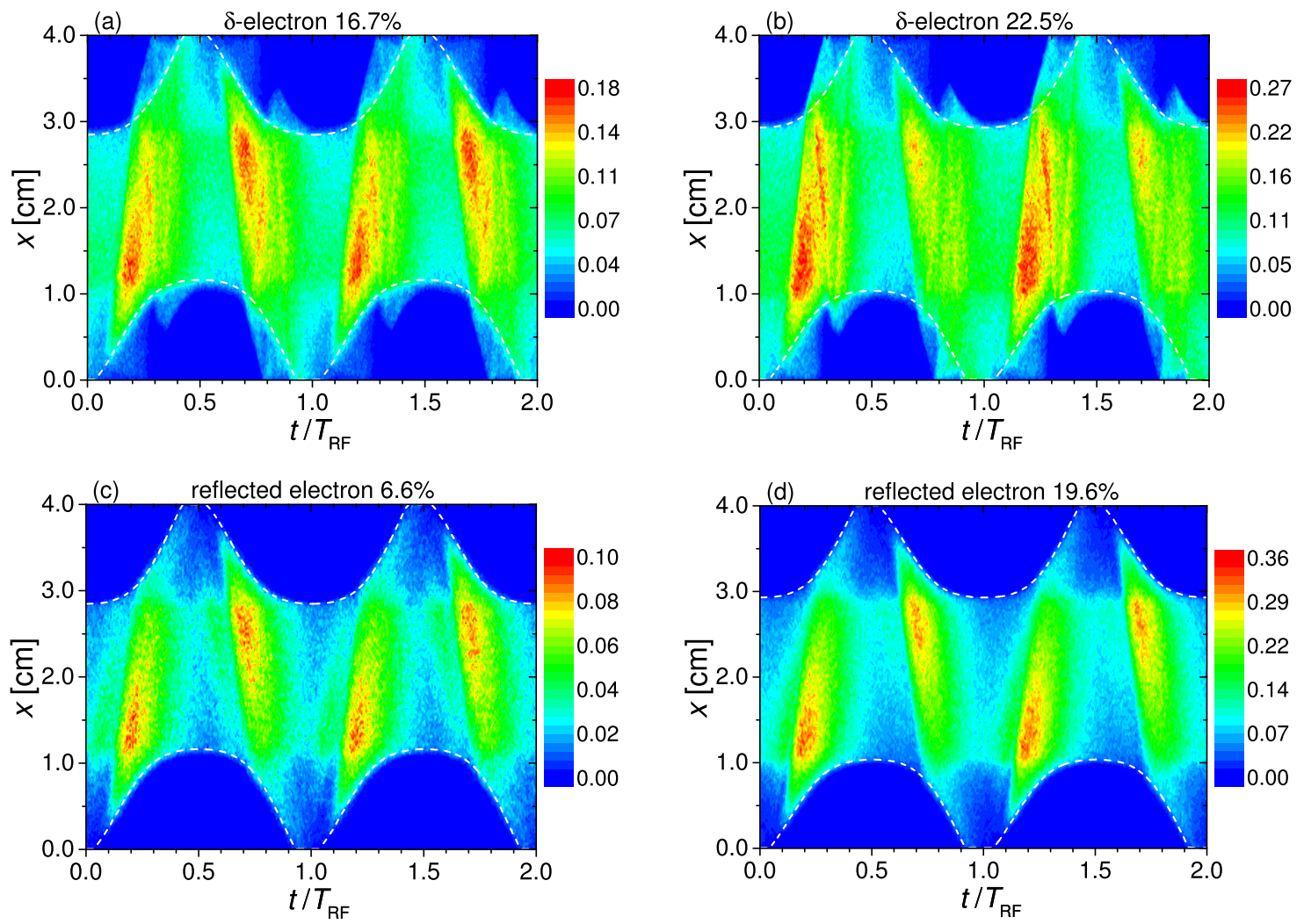

FIG. 7. Spatiotemporal distributions of the contributions of $\delta$-electrons (top row) and reflected electrons (bottom row) to the total ionization (shown in Fig. 5) obtained from case A (left column) and case B (right column). Discharge conditions: $d=4.0 \mathrm{~cm}, f=13.56 \mathrm{MHz}, p=2.0 \mathrm{~Pa}$, and $\mathrm{V}_{0}=1000 \mathrm{~V}$. The sheath edges are defined by $\left|E_{z}\right|=5000 \mathrm{Vm}^{-1}$. The color bars are given in units of $10^{21} \mathrm{~m}^{-3} \mathrm{~s}^{-1}$. 
Fig. 9(d)], which leads to an asymmetric distribution of ionization induced by $\delta$-electrons in case B. Meanwhile, a competitive contribution between the reflected electrons and the $\delta$-electrons to the ionization rate has been observed in case B. Despite the fact that the maximum of the elastic reflection of the powered electrode $\left(\eta_{\mathrm{el}, \text {,max }}\right.$ $=0.5)$ is much higher than that of the grounded electrode $\left(\eta_{\mathrm{el}, \mathrm{max}}\right.$ $=0.1$ ), the contribution of reflected electrons emitted from two electrodes is approximately identical in case B [shown in Fig. 7(d)]. The $\delta$-electrons accelerated by residual sheath voltage near the powered electrode can gain more energy compared to the reflected electrons. As a result, the $\delta$-electrons dominate the ionization dynamics and consequently contribute a significant ionization rate.

To have deep insight into the asymmetric effects induced by different material electrodes, the composition of the ionization rate contributed by $\sigma$-electrons emitted from their original electrode obtained from case A (top row) and case B (bottom row) is illustrated in Fig. 8. It is shown that the ionization rate caused by $\sigma$-electrons emitted from both electrodes is symmetric in case A (top row). However, an asymmetric contribution of $\sigma$-electrons to the ionization rate is observed in case B (bottom row). $\sigma$-electrons emitted by the powered electrode contribute about 3 times higher ionization rates than those of SEs emitted by the grounded electrode.

The incident electron (In. $\mathrm{e}^{-}$) flux and outgoing electron (Out. or $\sigma \mathrm{e}^{-}$) flux, at the powered (bottom-left scale) and the grounded (top-right scale) electrodes for both cases, are shown in the top row of Fig. 9. In case A, the flux of In. or Out. $\mathrm{e}^{-}$at both sides of the electrode is symmetric. While in case B, both the In. and Out. $\mathrm{e}^{-}$fluxes at the powered electrode are higher than those at the grounded electrode, indicating that the contribution of $\sigma$-electrons emitted from the powered electrode to the ionization rate is higher than that emitted from the grounded electrode [see Fig. 6(d)]. Moreover, the total flux of SEs is slightly higher than that of In. electrons in the phase about $0.8<t / T_{\mathrm{RF}}<0.9$ and $1.1<t / T_{\mathrm{RF}}<1.3$, within which the sheath has not completely collapsed at the powered electrode for $V_{0}=1000 \mathrm{~V}$ in case $\mathrm{B}$. This reveals that the emission of electron-induced $\delta$-electrons is significant because of high SEECs in these phases. However, the In. $\mathrm{e}^{-}$flux is always lower than the flux of Out. $\mathrm{e}^{-}$near the Cu electrode in both cases due to lower SEECs.

The fluxes of reflected electrons and $\delta$-electrons at the powered (bottom-left scale) and the grounded (top-right scale) electrodes for both cases are shown in the bottom row of Fig. 9. There is an obviously different emission behavior between reflected and $\delta$-electrons. As can be seen, the Out. flux of reflected electrons emitted at two electrodes is higher than the Out. flux of $\delta$-electrons because a lot of low energy bulk electrons can reach the electrode when the sheath is completely collapsed (e.g., the phase of $0.9<t / T_{\mathrm{RF}}<1.1$ near the powered electrode) and then be reflected. We can also see that part of the $\delta$-electrons emitted before the sheath is completely collapsed (e.g., the phase of $0.8<t / T_{\mathrm{RF}}<0.9$ near
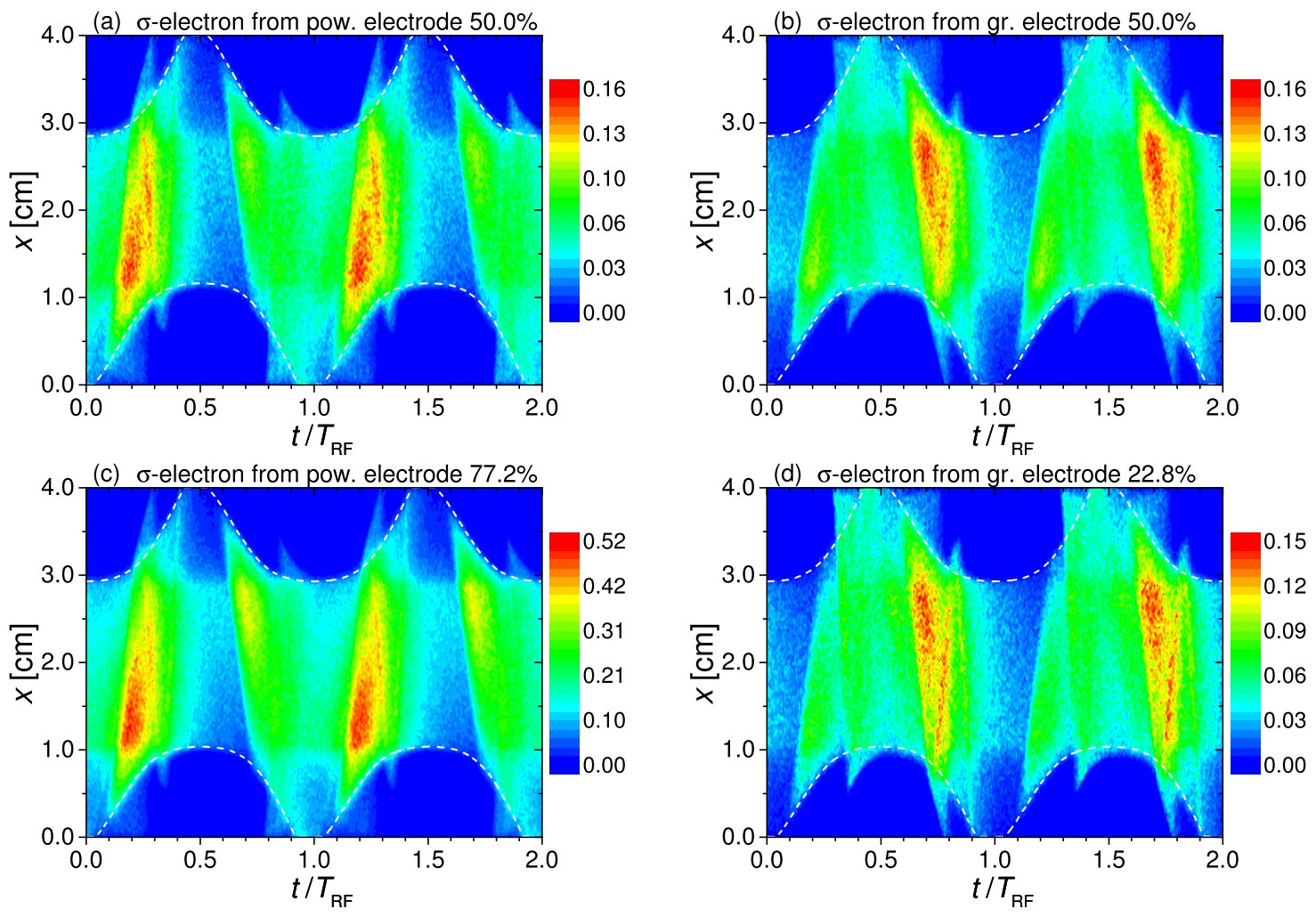

FIG. 8. Spatiotemporal distributions of the ionization rate induced by $\sigma$-electrons emitted at the powered (left column) and the grounded (right column) electrode, obtained from case $\mathrm{A}$ (top row) case $\mathrm{B}$ (bottom row). Discharge conditions: $d=4.0 \mathrm{~cm}, f=13.56 \mathrm{MHz}, p=2.0 \mathrm{~Pa}$, and $\mathrm{V}_{0}=1000 \mathrm{~V}$. The sheath edges are defined by $\left|E_{z}\right|=5000 \mathrm{~V} \mathrm{~m}^{-1}$. The color bars are given in units of $10^{21} \mathrm{~m}^{-3} \mathrm{~s}^{-1}$. 

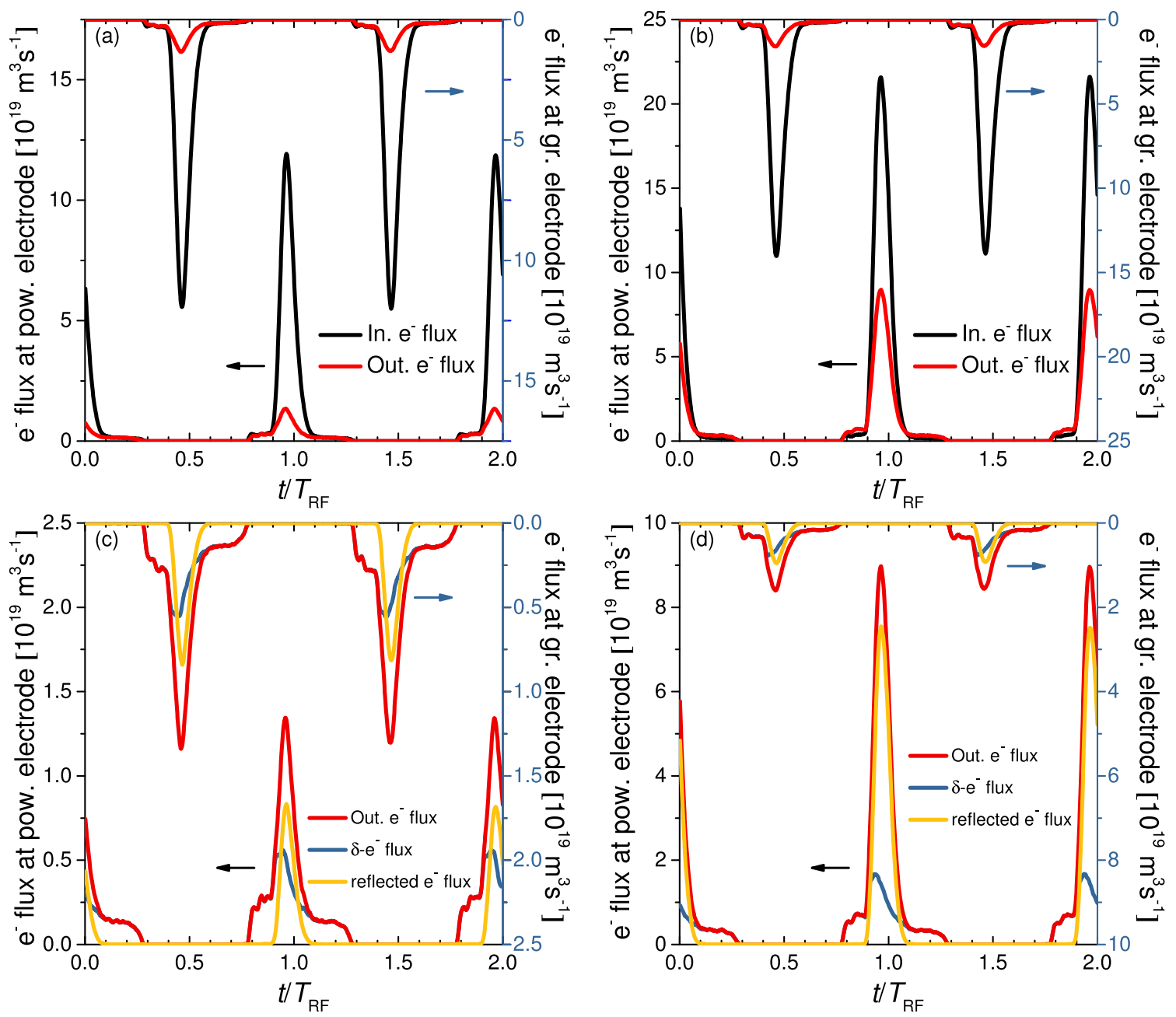

FIG. 9. Incident electron flux (In. $\mathrm{e}^{-}$) and electron-induced total SEs (which comprises all the three components of electron-induced SEs) flux (Out. or $\sigma$ e $\mathrm{e}^{-}$(top row) and outgoing electrons and their components $\left(\delta-\mathrm{e}^{-}\right.$and reflected $\left.\mathrm{e}^{-}\right)$(bottom row) at the powered (bottom-left scale) and the grounded (top-right scale) electrodes obtained from case $\mathrm{A}$ (left column) and case B (right column), respectively. Discharge conditions: $d=4.0 \mathrm{~cm}, f=13.56 \mathrm{MHz}, p=2.0 \mathrm{~Pa}$, and $V_{0}=1000 \mathrm{~V}$.

the powered electrode), which are accelerated by residual sheath potential. Hence, the energy of the $\delta$-electron beam is high enough to traverse the entire bulk and bounced back by the opposite sheath, which then forms the trajectory of $\delta$-electron beams, as clearly seen in the top row of Fig. 7. Although the flux of reflected electrons is greater than the flux of $\delta$-electrons, $\delta$-electrons contribute a higher ionization rate compared to the reflected electrons.

The propagating path of highly energetic SEs emitted from electrodes is presented in Fig. 10 which shows spatiotemporal density distributions of $\sigma$-electrons (first row), $\delta$-electrons (second row), and reflected electrons (third row) with the energy above $15 \mathrm{eV}$ obtained from case A (left column) and case B (right column). The threshold $\varepsilon_{0}$ $=15 \mathrm{eV}$ for electron-induced $\delta$-electron emission is close to the threshold $\varepsilon_{i}=15.8 \mathrm{eV}$ for electron-impact ionization of the background gas. Thus, the spatiotemporal density distributions of energetic SEs are similar to the spatiotemporal distributions of the ionization rate induced by SEs. The highly energetic beams of SEs accelerated by the expanding sheath near the local electrode hit the opposite electrode in the phase, within which the residual sheath voltage is still high enough to bounce most of these SEs back to the plasma bulk. This leads to an effective confinement of these energetic electrons in the bulk and causes a high ionization (see Fig. 7). A beam of reflected electrons generated in the collapse phase (e.g., the phase of $0.9<t / T_{\mathrm{RF}}$ $<1.1$ near the powered electrode as shown in the bottom row of Fig. 9) is clearly visible as shown in the bottom row of Fig. 10. This beam is caused by the emission of the high energy portion of reflected electrons without sheath acceleration. The driving voltages can influence the fluxes and energies of ions and electrons at the electrodes and hence affect the ionization dynamics. Figure 11 shows the ionization proportion of $\gamma_{-}, \delta$-, reflected, and bulk electrons as a function of $V_{0}$ obtained from case A [shown in Fig. 11(a)] and case B [shown in Fig. 11(b)]. Under the present conditions, the bulk electron beam is generated at the expanding sheath edge, which propagates through the bulk and dominates ionization for both cases. The most 

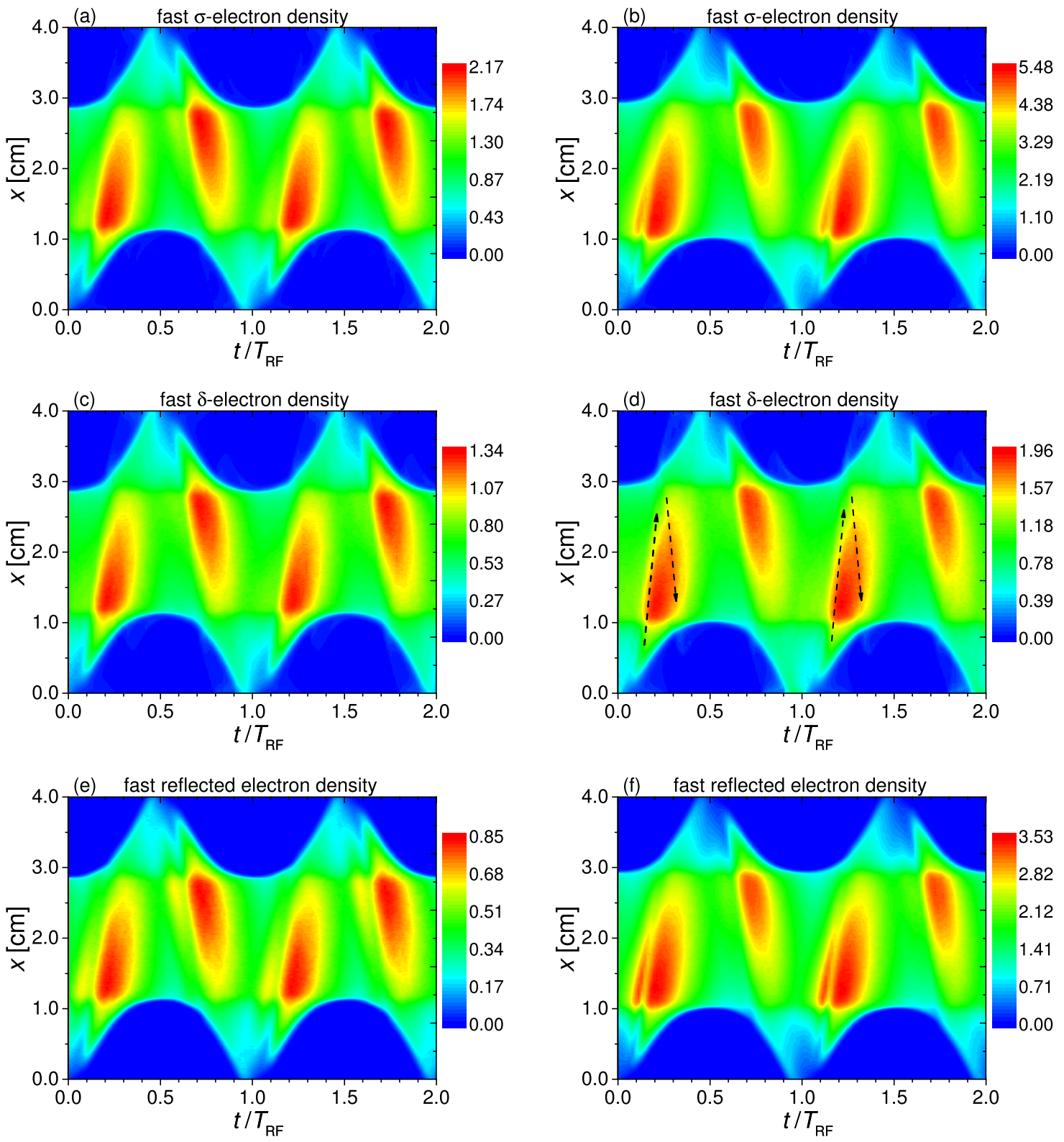

FIG. 10. Spatiotemporal distributions of the density of $\sigma$-electrons (first row), $\delta$-electrons (second row), and reflected electrons (third row) with energies above $15 \mathrm{eV}$ obtained from case A (left column) and case B (right column). Discharge conditions: $d=4.0 \mathrm{~cm}, f=13.56 \mathrm{MHz}, p=2.0 \mathrm{~Pa}$, and $V_{0}=1000 \mathrm{~V}$. The color bars are given in units of $10^{13} \mathrm{~m}^{-3}$.

significant portion of the ionization rate is directly generated by bulk electrons in case $\mathrm{A}$ at low voltages and maintains above $60 \%$ of the total ionization rate at $V_{0} \geqslant 200 \mathrm{~V}$. However, as $V_{0}$ increases, the contribution of bulk electrons to the total ionization rate accordingly decreases gradually, and the ionization proportion reduces to about $47 \%$ at $V_{0}=2000 \mathrm{~V}$ in case B. Note that only the contribution of $\delta$-electrons to the ionization increases with increasing $V_{0}$ due to energy-dependent SEECs in both cases. In case B, the ionization proportion of $\delta$-electrons is lower than that of reflected electrons at relatively low driving voltages $\left(V_{0}<800 \mathrm{~V}\right)$. Upon further increasing $V_{0}$, the ionization proportion of $\delta$-electrons continues to increase and finally exceeds the ionization proportion of reflected electrons. This trend reveals that the influence of electroninduced SEs in the ionization dynamics cannot be ignored at low pressures, especially at high driving voltages in CCPs. Different from case A, the ionization proportion of reflected electrons is always higher than that of $\gamma$-electrons in case B. Therefore, the contribution of reflected electrons to the ionization rate is also pronounced when the maximum value of the elastic reflection $\eta_{\mathrm{el} . \text {,max }}$ is high. 

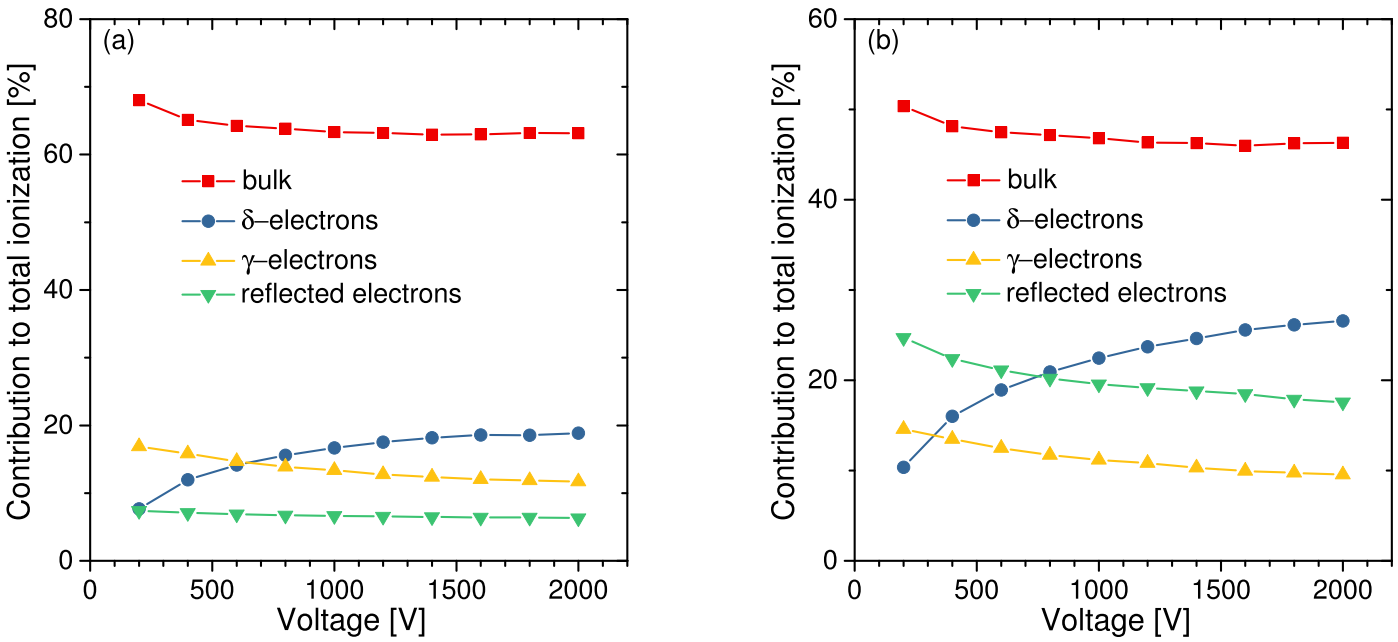

FIG. 11. The individual contribution of $\gamma$-, $\delta$-, reflected, and bulk electrons to the total ionization rate obtained from case $\mathrm{A}(\mathrm{a})$ and case $\mathrm{B}$ (b) as a function of the driving voltage amplitude, $V_{0}$. Discharge conditions: $d=4.0 \mathrm{~cm}, f=13.56 \mathrm{MHz}$, and $p=2.0 \mathrm{~Pa}$.

\section{CONCLUSIONS}

In this paper, a realistic model, describing electron-surface interactions including the elastic reflection of electrons, the inelastic backscattering of electrons, and the emission of true electron-induced SEs as a function of the incident electron energy and angle, is implemented in PIC/MCC simulations. With this model, we have studied the effects of asymmetric SEE induced by different electrode materials in lowpressure argon CCPs. The discharges are operated at a low pressure of $2.0 \mathrm{~Pa}, f=13.56 \mathrm{MHz}$, and over a wide range of driving voltages from 200 to $2000 \mathrm{~V}$ in argon discharges. Considering the practical application, two different materials are considered for the powered electrode, i.e., $\mathrm{Cu}$ and $\mathrm{SiO}_{2}$ electrodes considered as cases $\mathrm{A}$ and $\mathrm{B}$, respectively. The results obtained from simulations based on case B were compared with those obtained from case A to comprehensively understand the electron-surface interactions. A pronounced difference of SE flux induced by asymmetric electrode materials is observed, which leads to asymmetric plasma characteristics and ionization dynamics.

The simulation results in terms of spatiotemporal ionization distributions of the different groups of electrons $(\gamma-, \delta$-, reflected, and bulk electrons) generated at the powered and grounded electrode were presented separately. Because of multiple reflections of energetic ioninduced and electron-induced SEs between two oscillating sheaths, highly energetic $\delta$-electrons contribute significantly to ionization, especially at high driving voltages in case B. Furthermore, field reversals are observed in the phases when sheaths completely collapse at the powered electrode at high driving voltage amplitudes due to high SEECs of $\mathrm{SiO}_{2}$.

In general, these results show that the effects of asymmetric SEE induced by different material electrodes are essential at low pressures and high driving voltages. However, it is difficult to realize effective heating of highly energetic electrons in the case of the SF discharge investigated here. It is expected that the influence of electron-induced SEs on the discharge characteristics could be pronounced in dualfrequency discharges, where the conditions of collisionless bounceresonance heating ${ }^{40}$ are relatively easy to be satisfied. Besides, $\gamma$ - electrons generated by ions have a significant contribution to the emission of $\delta$-electrons at low pressures. ${ }^{23}$ The $\gamma$-electron yield largely depends on the properties of the electrode surfaces. These $\gamma$-electrons are expected to be heated by the sheath at the DC electrode and achieve very high energy due to the DC voltage. The combination of the realistic model for the electron-induced SEE with energydependent SE yields due to the heavy particle impact in DC superposed RF discharge will be discussed in the future work.

\section{ACKNOWLEDGMENTS}

This work was financially supported by the National Natural Science Foundation of China (NSFC) (Grant Nos. 11335004, 11675039,11722541 , and 11875100).

\section{REFERENCES}

${ }^{1}$ T. Makabe and Z. L. Petrovic, Plasma Electronics: Applications in Microelectronic Device Fabrication (CRC Press, 2014), Vol. 26.

${ }^{2}$ P. Chabert and N. Braithwaite, Physics of Radio-Frequency Plasmas (Cambridge University Press, 2011).

${ }^{3}$ J. Musil, Physical and Mechanical Properties of Hard Nanocomposite Films Prepared by Reactive Magnetron Sputtering (Springer, New York, 2006).

${ }^{4}$ J. P. Verboncoeur, "Particle simulation of plasmas: Review and advances," Plasma Phys. Controlled Fusion 47(5A), A231 (2005).

${ }^{5}$ C. K. Birdsall and A. B. Langdon, Plasma Physics via Computer (McGraw-Hill, Inc., 1985).

${ }^{6}$ C. K. Birdsall, "Particle-in-cell charged-particle simulations, plus monte carlo collisions with neutral atoms, pic-mcc," IEEE Trans. Plasma Sci. 19(2), 65-85 (1991).

7Z. Donkó, "Particle simulation methods for studies of low-pressure plasma sources," Plasma Sources Sci. Technol. 20(2), 24001-24015 (2011).

${ }^{8}$ J. Schulze, Z. Donkó, B. G. Heil, D. Luggenhölscher, T. Mussenbrock, R. P. Brinkmann, and U. Czarnetzki, "Electric field reversals in the sheath region of capacitively coupled radio frequency discharges at different pressures," J. Phys. D: Appl. Phys. 41(10), 105214 (2008).

${ }^{9}$ Z. Donkó, J. Schulze, P. Hartmann, I. Korolov, U. Czarnetzki, and E. Schüngel, "The effect of secondary electrons on the separate control of ion energy and 
flux in dual-frequency capacitively coupled radio frequency discharges," Appl. Phys. Lett. 97(8), 81501 (2010).

${ }^{10}$ J. Schulze, Z. Donkó, E. Schüngel, and U. Czarnetzki, "Secondary electrons in dual-frequency capacitive radio frequency discharges," Plasma Sources Sci. Technol. 20(4), 45007 (2011).

"ZZ. Donkó, J. Schulze, U. Czarnetzki, A. Derzsi, P. Hartmann, I. Korolov, and E. Schüngel, "Fundamental investigations of capacitive radio frequency plasmas: Simulations and experiments," Plasma Phys. Controlled Fusion 54(12), 124003 (2012).

${ }^{12}$ Y. X. Liu, Q. Z. Zhang, W. Jiang, W. Q. Lu, and Y. N. Wang, "Experimental validation and simulation of collisionless bounce-resonance heating in capacitively coupled radio-frequency discharges," Plasma Sources Sci. Technol. 21(3), 035010 (2012)

${ }^{13}$ Q.-Z. Zhang, Y.-X. Liu, W. Jiang, A. Bogaerts, and Y.-N. Wang, "Heating mechanism in direct current superposed single-frequency and dual-frequency capacitively coupled plasmas," Plasma Sources Sci. Technol. 22, 025014 (2013).

${ }^{14}$ A. Derzsi, I. Korolov, E. Schüngel, Z. Donkó, and J. Schulze, "Electron heating and control of ion properties in capacitive discharges driven by customized voltage waveforms," Plasma Sources Sci. Technol. 22(6), 065009 (2013).

${ }^{15}$ O. Braginsky, A. Kovalev, D. Lopaev, O. Proshina, T. Rakhimova, A. Vasilieva, D. Voloshin, and S. Zyryanov, "Experimental and theoretical study of dynamic effects in low-frequency capacitively coupled discharges," J. Phys. D: Appl. Phys. 45(1), 015201 (2012).

${ }^{16}$ J. Schulze, A. Derzsi, I. Korolov, E. Schuengel, and Z. Donko, "Electron heating and control of ion properties in capacitive discharges driven by customized voltage waveforms," Plasma Sources Sci. Technol. 22(6), 065009 (2013).

${ }^{17}$ A. Derzsi, I. Korolov, E. Schüngel, Z. Donkó, and J. Schulze, "Effects of fast atoms and energy-dependent secondary electron emission yields in PIC/MCC simulations of capacitively coupled plasmas," Plasma Sources Sci. Technol. 24(3), 34002 (2015).

${ }^{18} \mathrm{H}$. Hannesdottir and J. T. Gudmundsson, "The role of the metastable O2(b1 $\left.\Sigma+\mathrm{g}\right)$ and energy-dependent secondary electron emission yields in capacitively coupled oxygen discharges," Plasma Sources Sci. Technol. 25(5), 055002 (2016).

${ }^{19}$ M. Daksha, A. Derzsi, S. Wilczek, J. Trieschmann, T. Mussenbrock, P. Awakowicz, Z. Donkó, and J. Schulze, "The effect of realistic heavy particle induced secondary electron emission coefficients on the electron power absorption dynamics in single- and dual-frequency capacitively coupled plasmas," Plasma Sources Sci. Technol. 26(8), 085006 (2017).

${ }^{20}$ M. Daksha, A. Derzsi, Z. Mujahid, D. Schulenberg, and B. Berger, "Material dependent modeling of secondary electron emission coefficients and its effects on PIC/MCC simulation results of capacitive RF plasmas," Plasma Sources Sci. Technol. 28(3), 034002 (2019).

${ }^{27}$ V. P. Gopinath, J. P. Verboncoeur, and C. K. Birdsall, "Multipactor electron discharge physics using an improved secondary emission model," Phys. Plasmas 5(5), 1535-1540 (1998).

${ }^{22}$ B. Horváth, M. Daksha, I. Korolov, A. Derzsi, and J. Schulze, "The role of electron induced secondary electron emission from $\mathrm{SiO}_{2}$ surfaces in capacitively coupled radio frequency plasmas operated at low pressures," Plasma Sources Sci. Technol. 26(12), 124001 (2017).

${ }^{23}$ B. Horváth, J. Schulze, Z. Donkó, and A. Derzsi, “The effect of electron induced secondary electrons on the characteristics of low-pressure capacitively coupled radio frequency plasmas," J. Phys. D: Appl. Phys. 51(35), 355204 (2018).

${ }^{24}$ I. Korolov, A. Derzsi, Z. Donkó, and J. Schulze, "The influence of the secondary electron induced asymmetry on the electrical asymmetry effect in capacitively coupled plasmas," Appl. Phys. Lett. 103(6), 64102 (2013).

${ }^{25}$ T. Lafleur, P. Chabert, and J. P. Booth, "Secondary electron induced asymmetry in capacitively coupled plasmas," J. Phys. D: Appl. Phys. 46(13), 135201 (2013).

${ }^{26}$ J. P. Verboncoeur, M. Virginia Alves, V. Vahedi, and C. Kennedy Birdsall, "Simultaneous potential and circuit solution for 1d bounded plasma particle simulation codes," J. Comput. Phys. 104(2), 321-328 (1993).

${ }^{27}$ C. K. Birdsall and A. B. Langdon, Plasma Physics via Computer Simulation (CRC Press, 2004).

${ }^{28}$ A. V. Phelps and Z. L. Petrovic, "Cold-cathode discharges and breakdown in argon: Surface and gas phase production of secondary electrons," Plasma Sources Sci. Technol. 8(3), R21 (1999).

${ }^{29} \mathrm{~V}$. Vahedi and M. Surendra, "A monte carlo collision model for the particle-incell method: Applications to argon and oxygen discharges," Comput. Phys. Commun. 87(1-2), 179-198 (1995).

${ }^{30} \mathrm{Q}$. Z. Zhang, W. Jiang, L. J. Hou, and Y. N. Wang, "Numerical simulations of electrical asymmetry effect on electronegative plasmas in capacitively coupled rf discharge," J. Appl. Phys. 109(1), 013308 (2011).

${ }^{31}$ D. Sydorenko, "Particle-in-cell simulations of electron dynamics in low pressure discharges with magnetic fields," Ph.D. thesis (University of Saskatchewan, 2006).

${ }^{32}$ J. Rodney and M. Vaughan, "A new formula for secondary emission yield," IEEE Trans. Electron Devices 36(9), 1963-1967 (1989).

${ }^{33}$ R. Vaughan, "Secondary emission formulas," IEEE Trans. Electron Devices 40(40), 830 (1993).

${ }^{34}$ S. Barral, K. Makowski, Z. Peradzyński, N. Gascon, and M. Dudeck, "Wall material effects in stationary plasma thrusters. ii. near-wall and in-wall conductivity," Phys. Plasmas 10(10), 4137-4152 (2003).

${ }^{35} \mathrm{H}$. Seiler, "Secondary electron emission in the scanning electron microscope," J. Appl. Phys. 54(11), R1-R18 (1983).

${ }^{36}$ I. M. Bronshtein and B. S. Fraiman, Vtorichnaya elektronnaya emissiya (Secondary Electron Emission) (Atomizdat, Moscow,, 1969).

${ }^{37}$ M. A. Lieberman and A. J. Lichtenberg, Principles of Plasma Discharges and Materials Processing (John Wiley and Sons, 2005).

${ }^{38}$ J. Schulze, Z. Donkó, A. Derzsi, I. Korolov, and E. Schuengel, "The effect of ambipolar electric fields on the electron heating in capacitive RF plasmas," Plasma Sources Sci. Technol. 24(1), 15019 (2015).

${ }^{39}$ M. D. Campanell, A. V. Khrabrov, and I. D. Kaganovich, "Absence of debye sheaths due to secondary electron emission,” Phys. Rev. Lett. 108(25), 255001 (2012).

${ }^{40}$ Y. X. Liu, Q. Z. Zhang, W. Jiang, L. J. Hou, X. Z. Jiang, W. Q. Lu, and Y. N. Wang, "Collisionless bounce resonance heating in dual-frequency capacitively coupled plasmas," Phys. Rev. Lett. 107(5), 055002 (2011). 